\title{
Exposure to environmental chemical contaminants and infant diseases: constant hazard?
}

\begin{abstract}
The exposure to environmental chemical contaminants: lead, mercury, agrochemicals, PCBs, dioxins and BPA are characterized by situations of constant danger to healthy child development. Such substances exert toxic effects on multiple systems and organs, but scientific looks have been directed toward hormonal and neurobehavioral changes in childhood. The physiological needs and behavioral characteristics of the child raise the risk of childhood and adult disease from the exposure to these pollutants. Thus, this study made a brief review on the major toxic chemical contaminants, routes and forms of exposure and their possible associations with diseases in childhood. It has been observed that in recent decades, there have been few scientific advances, especially in emerging countries, regarding the understanding of the mechanisms involving the toxicity of one or multiplex biotics in low doses during the critical stages of development of the human organism. Further studies and efforts are suggested for the applicability of preventive measures and monitoring of indicators in children's environmental health by managers, health professionals and researchers around the world with the objective of reducing the risks of exposure of the child to environmental pollutants, in addition to diagnose and monitor health problems arising from this dangerous situation to the human life.
\end{abstract}

Keywords: environmental pollutants, child health, vulnerability, environmental health children
Volume 3 Issue 4 - 2017

\author{
Marcela de Abreu Moniz, Rayssa Bravo de \\ Oliveira Vollmer \\ Department of Nursing, Fluminense Federal University, Brazil
}

Correspondence: Marcela de Abreu Moniz, Department of Nursing, Institute of Humanities and Health, Fluminense Federal University, Rio das Ostras, Rio de Janeiro, Brazil,Tel 552198810806I, Email marceladeabreumoniz@gmail.com

Received: August 20, 2017 | Published: August 24, 2017

\section{Introduction}

The environmental chemical exposure is directly related to the risk of developing diseases at all stages of human life. However, childhood is a particularly vulnerable stage to exposure to various environmental contaminants and their effects of toxicity on various organs and systems. Around the world, about three million children under five years old die each year from diseases associated with environmental pollution. ${ }^{1,2}$ The child behavioral factor makes children a group extremely susceptible to contact with different environmental chemical contaminants, such as crawling, putting objects into the mouth, playing near the ground. The physiological demands of children also accentuate the health risks of environmental chemical exposure due to an increased water and food demand, the lower body weight, and the greater amount of air breathed per unit of weight when compared to an adult. ${ }^{1,3}$ In the last decades, the contamination of the environment by chemical agents resulting from industrial activities, mineral exploration and agricultural production and, similarly, the increase in the prevalence of congenital malformations, asthma, cancer, neurological and behavioral disorders in children has raised great concern in populations and scientists. ${ }^{3,4}$

Considering that the organism of the fetus, the newborn, and the child presents its physiological systems in the process of maturation, the acute and chronic exposure to toxic chemicals in these critical stages of development can cause reversible and irreversible damages to their physical and mental health. In this context, another worrying and quite current fact is the lack of scientific knowledge and its applicability in the area of neonatal and child healthcare about the mechanisms of action of persistent organic pollutants (pesticides, Dioxins and furanospoliclorates, biphenylspolyichlorates) and heavy metals (lead; mercury) as endocrine disrupters. These are characterized in exogenous chemical substances capable of producing health effects resulting from the hormonal changes in the human organism. The neuroendocrine effects resulting from the exposure to endocrine disrupters during fetal, perinatal or infantile life may imply greater long-term risks to the individual's life. ${ }^{5,6}$ Thus, this study made a brief review on the major toxic chemical contaminants, routes and forms of environmental exposure and their possible associations with diseases in childhood.

\section{Discussion}

\section{Environmental chemical pollutants: exposure standards and toxic health effects on children}

Mercury: Mercury is a heavy metal that has a complex biogeochemical nature and its natural cycle in soil, water, and air has been profoundly modified by anthropic activities. Inorganic forms of mercury are used in the production of silver amalgam in dental restoration (mercury alloy with silver), tools (thermometers and sphygmomanometers), medicines, cosmetics, and other industrial processes. The inadequate disposal of industrial waste with organic mercury compounds causes sedimentation in the water and, later, the methylation process by sulphate $\left(\mathrm{SO}_{4}\right)$ processing bacteria, thus transforming it into the organic form that contaminates plankton, plants, sea animals, and rivers. In the Amazon region, although mining is an important source of mercury contamination, the main source comes from fires that release a large amount of inorganic mercury present in the soil and makes it available to the aquatic system, where the processes of bio methylation and bioaccumulation occur. The consumption of food of marine origin exposes the man to the inorganic compounds of mercury. Inorganic species (methyl, ethyl, and dimethyl mercury) are about 100 to 1000 more toxic to humans than organic species. ${ }^{4}$ Inorganic forms are also used in several industrial processes (industries of plastics, paper, medicines, paints, pesticides, wood preservatives, and 
petrochemicals). ${ }^{7}$ Human exposure to mercury particles or residues can generate toxic effects on the kidneys, central nervous system, liver, heart, lungs, and DNA. As a volatile compound, mercury can be inhaled by children, causing them to compromise the lung function and increase the number of hospitalizations for acute respiratory infections. ${ }^{4}$ This situation is aggravated because children generally inhale a greater amount of air pollutants than adults due to the need for inhalation of a higher respiratory volume per minute, per kilogram of body weight, as a result of their increased basal metabolism. ${ }^{8}$ The methylmercury may interfere in one or more of the critical stages of development of the nervous system (proliferation, migration, differentiation, synapse, myelination to apoptosis) causing temporary or irreversible damage to the cognitive, sensory and motor functions. The genotoxic and teratogenic effects of fetal intrauterine exposure to methylmercury may be evidenced by congenital anomalies in the central nervous system such as microcephaly and major mental retardation. ${ }^{7}$ Such a toxic substance may cross the placental and the blood-brain barrier; and the fetal sensitivity is 3 to 4 times greater than that of the adult. Other forms of infant exposure to methylmercury include breastfeeding and the ingestion of food and water with a contaminated hand. ${ }^{1}$ Mercury and other heavy metals such as lead may lead to acute renal failure in the child as they may impair the glomerular filtration function of salts, liquids, and blood residues, resulting in local hydro electrolyte imbalance. In addition, the excretion capacity of these substances is much smaller than in adults, causing these substances to remain in the body for a longer period. ${ }^{4}$

Lead: Lead is another heavy metal accumulating in organisms, whose toxic potential can damage any biological activity and compartment. ${ }^{9}$ This xenobiotic produces toxic effects on the peripheral nervous system and on the renal system of adults, and on the central nervous system of children, as well as other effects such as hypochromic microcytic anemia, hypertension, anorexia, reduced fertility, reduced physical growth and height in children. ${ }^{7}$ Lead can cause clinical, subclinical, or only biochemical effects. In recent decades, clinical and epidemiological studies on the neurotoxic effects in children have contributed to the reduction of the tolerable limits of lead in the blood of this susceptible group. Low levels of exposure to this toxic element may be able to impair the neuropsychocognitive development of children, since the dose-response relationship is not accurate. In addition, in childhood, the toxicity effects of lead may reduce the intelligence, and the psychomotor and neurobehavioral disorders may be permanent. Chronic exposure to lead may affect the hormone levels of gonadotrophins and bone development, although it is deposited in larger amounts in the bones of adults than in children. However, this fact causes more lead to be circulating and available to exert its toxic effects on the different tissues: nervous, renal, bone, hematological, cardiovascular, and reproductive. ${ }^{1,9}$ The widespread use of lead in metallurgy and the manufacture of paints, pigments, and batteries results in the persistence of this pollutant in the soil, water and air, posing a risk of exposure by the respiratory and gastrointestinal tract. ${ }^{7}$ In addition, other environmental factors exacerbate the risks of chemical exposure in childhood. Groups of children with unfavorable socioeconomic conditions living in urban or industrial areas generally have higher levels of lead in their blood and urine when compared to groups of children with better living conditions and/or dwellings that are located far from industrial plants. ${ }^{1,10}$

Dioxins, polychlorinatedbiphenyls (PCBS), bisphenol A(BPA): The PCBs, BPA, and dioxins are highly toxic and persistent chemical compounds in the environment and living organisms that can generate chronic adverse effects on ecosystems and human health. The PCBs are a group with 209 congeners and their use reduction in the composition of plastics and other materials (oils, insulation, paints) date from the 70's. ${ }^{6}$ Dioxins is a term used to denote a set of compounds with a chemical class known as polychlorinated dibenzop-dioxins (PCDDs) and dibenzofuranspolychlorates (PCDFs), which are subproducts obtained from the synthesis of some organic materials (polyvinyl chloride - PVC -, some pesticides such as herbicides: 2,4-D; 2,4,5-T alachlor and organochlorine insecticides: chlordane, dieldrin, DDT and metabolites; paper); or from the uncontrolled incineration of organic waste or mixtures containing chlorine. ${ }^{5}$ There is evidence that some PCBs exert similar toxicity to 2,3,7,8-tetrachlorodibenzop-dioxin (TCDD), which is the most toxic congener of dioxins. The BPA is a synthetic monomer used to produce large-scale industrial polycarbonate plastics around the world. Thus, the BPA is an essential component of water bottles, containers, bottles, epoxy resins, which are used to varnish food cans, PVC, sealants, dental resins, thermal paper, paints, and catheters for medical use..$^{11}$ The environmental exposure to the toxic compounds Dioxins, PCBS and BPA can occur through the inhalation, dermal, and digestive routes, since the contamination by these substances is widely disseminated by environmental matrices. Because they are lipophilic substances, they are poorly absorbed in the gastrointestinal tract in the first months of age due to the physiological immaturity of the enter hepatic circulation of bile acids and their metabolic functions. However, breast milk is an important source of exposure of these substances to the breastfed infant. There is greater distribution of these compounds to the organs and less amount is deposited in the adipose tissue. ${ }^{1,4}$ The BPA can promote epigenetic, estrogenic, and neurobehavioral changes. ${ }^{11}$ The PCBs are considered immunotoxic and they may induce changes in the T-dependent cell's response and favor the emergence of viral, autoimmune, allergic, and cancer in childhood and adulthood. In addition, they stimulate the estrogen receptors and cause the blockade of thyroxine receptors and may lead to cognitive changes. ${ }^{6} 12$ The dioxins act as estrogen receptor antagonists. Thus, dioxins, BPA, as well as organochlorines, can trigger signs of early puberty. Other pathologies may be generated by hormonal imbalance caused by BPA, PCBs, and dioxins, such as: breast cancer, male infertility, polycystic ovarian syndrome, prostate hyperplasia/cancer, genitalia changes, and early telarch. ${ }^{6}$

Pesticides: The indiscriminate agricultural and domestic use of agrochemicals can produce serious public health problems. The scientific knowledge is still limited on the effects on children s health from the exposure to low doses of different classes of pesticides. The main routes of child exposure are the digestive and inhalation. ${ }^{1}$ The blood-brain barrier is not fully developed until six months of age, which allows some toxic substances, such as pesticides and some metals, to cross the endothelial layer and affect the brain tissue. ${ }^{4}$ There is great concern regarding the organochlorine compounds that act as endocrine disrupters and are highly persistent and bio accumulative. Although most of these products have been withdrawn from the market due to their high toxicity to living beings for more than 20 years now, as it is the situation in Brazil, their metabolites are widely found in the human body, where they remain deposited in the fatty tissue due to human exposure, and they can be demobilized and released slowly into the bloodstream, producing chronic effects. ${ }^{5}$ Thus, children fed with breast milk can be exposed to organochlorine residues ${ }^{4}$. There is evidence of the interference of these compounds in estrogenic and androgenic actions and inhibition of the thyroid hormone synthesis. ${ }^{6}$ Immunotoxic and neurotoxic effects (cognitive and neurological 
impairment, behavioral changes) in children caused by the exposure to these environmental pollutants have also been reported in the literature. Currently, the most commonly used agrochemicals in the world are products of the organ phosphorus class. These inhibit the action of the acetylcholinesterase enzyme and may trigger neurotoxic and detrimental effects on children's cognitive development, such as hyperactivity and attention problems. ${ }^{1,12}$

\section{Conclusion}

This review ratified the need to develop strategies to monitor situations of constant danger to a healthy child development, which are their exposure to environmental chemical contaminants: lead, mercury, agrochemicals, PCBs, dioxins, and BPA. There are challenges in the professional, educational, scientific, and political fields for joint action in order to identify and mitigate the conditions and risk factors of the child exposure to environmental pollutants and to associate them with possible harmful effects on the health of children in different realities. The efforts should be greater in contexts that present socio-environmental determinants such as poverty, restricted access to health services, and lack of access to information on health promotion practices. ${ }^{4}$ To this end, new scientific information from clinical, toxicological, and epidemiological studies should be generated in order to understand the mechanisms of the toxic action of one or multiple xenobiotics in low doses during the critical phases of development of the human organism, since, in the last decades, there have been few scientific advances on this subject, especially in emerging countries. ${ }^{1}$ In addition, it is suggested that the appropriate use of the knowledge already acquired and its applicability in preventive measures and monitoring of indicators in children's environmental health is one of the proposals that need to be considered by managers, health professionals, and researchers worldwide.

\section{Acknowledgements}

This study is based on the scholarships received by the Nursing Tutorial Education Program of the Rio das Ostras campus of the Fluminense Federal University by the Brazilian Ministry of Education.

\section{Conflict of interest}

The author declares no conflict of interest.

\section{References}

1. Mazoto Ml, Filhote MIF, Câmara VM, et al. Children's environmental health: a review of proposals and perspectives. Cad Saúde Colet. 2011;19(1):41-50.

2. Sousa JS, Campos RT, Silva AF, et al. Estimating and analyzing the determinants of the reduction of the infant mortality rate in Brazil. RBEUR. 2016;10(2):140-155.

3. Mello-da-Silva CA, Fruchtengarten L. Environmental chemical risks to children's health. J Pediatr. 2005;81(5):205-211.

4. Perlroth NH, Branco CW. Current knowledge about environmental exposure in children during the sensitive developmental period. $J$ Pediatr. 2017;93(1):17-27.

5. Meyer A, Sarcinelli PN, Abreu-Villaça Y, et al. Agrochemicals and their action as endocrine disrupters. In: Peres F, Moreira JC, editors. Is it poison or is it medicine?: Pesticides, health and the environment. Brazil: Fiocruz; 2003. p. 101-118.

6. Castro-Correia C, Fontoura M. The influence of environmental exposure on endocrine disruptors in the growth and development of children and adolescents. Rev Port Endocrinol Diabetes Metab. 2015;10(2):186-192.

7. Ayangbenro AS, Olubukola OB. A new strategy for heavy metal polluted environments: A review of micriobial biosorbents. Int J Environ Res Public Health. 2017;14(1):94.

8. Arbex MA, Santos Ude P, Martins LC, et al. Air pollution and respiratory system. J Bras Pneumol. 2012;38(5):643-655.

9. Moreira FR, Moreira JC. The effects of lead on the human body and its significance for health. Rev Panam Salud Publica. 2004;15(2):119-129.

10. Needleman HL, Bellinger D. The health effects of low level exposure to lead. Annu Rev Publ Health. 1991;12:111-140.

11. Bernardo PEM, Navas SA, Murata LTF, et al. Bisphenol A: Review on its use in the food packaging, exposure and toxicity. Rev Inst Adolfo Lutz. 2015;74(1):1-11.

12. Polanska K, Jurewicz J, Hanke W. Review of current evidence on the impact of pesticides, polychlorinated biphenyls and selected metals on attention deficit/ hyperactivity disorder in children. Int J Occup Med Environ Health. 2013;26(1):16-38. 